\title{
CFD STUDY OF MASS TRANSFER IN SPACER FILLED MEMBRANE MODULE
}

\author{
Sharmina Hussain \\ Department of Mathematics and Natural Science \\ BRAC University, 66, Mohakhali C/A, Dhaka, Bangladesh. \\ E-mail:sharmina@bracuniversity.ac.bd
}

Received 19.6.2010

Accepted 16.7.2011

\begin{abstract}
The present paper is devoted to investigate the mass transfer in two dimensional spacer filled channels. Three different configurations of the cylindrical spacers are considered with different channel Reynolds number and mesh length. Different size and shape of formation of recirculation region, upstream and downstream of the spacers are closely observed. This recirculation regions have an important role in enhancing the mass transfer in the reattachment region. CFD simulations show that the submerged spacers have the highest efficiency with highest wall shear stress and mass transfer enhancement whilst the cavity spacer have the least performance.
\end{abstract}

Key words: Concentration polarization, Mass transfer enhancement, Reattachment length

\section{Introduction}

The membrane can be defined as an imperfect mass barrier between systems with which it is in contact, and allows a preferred transport of one component from the inlet flow into the product. The main advantages of membrane separation processes comprise their unique separation capacity, possible separation of substances without phase conversion, usually at ambient temperature. Membrane separation processes also have a relatively low energy consumption, actual energy requirements depend on the character of the separated mixture and the membrane material. Continuous and automated operation are also advantageous factors. Membrane separation technology is gaining increasingly wide spread use because of its adaptability to a large number of application streams. For example, cold sterilization of beverage and pharmaceuticals, cell harvesting, metal recovery as colloidal oxides or hydroxide, desalination of brackish water, removal of micropollutents etc. are some of the industrial applications of membrane separation processes. Membranes are currently a component of multi-well plates used for the synthesis and screening of novel molecules in biotechnology.

In membrane processes accumulation of rejected species near the feed side of the membrane (concentration polarization) causes reduction of permeate flux and deterioration of permeate quality. Accumulation of rejected species can be suppressed by creating back mixing from the membrane to the bulk of the liquid.

This paper describes the CFD model and provides a discussion of mass transfer of a spacer filled two dimensional channel. The effects of single and multiple filaments on the mass transfer is presented. Effects of the types of spacers, channel Reynolds number, 
mesh length on the flow field, mass transfer enhancement and pressure loss along the channel is discussed based on the numerical simulations of the flow field.

\section{Problem Description:}

Spacer geometry, the channel geometry, typical grid generated for numerical simulation, the methods of numerical modeling etc. has been depicted in details in [1]. The schematic representation of a net type spacer (Fig.1), different configuration of spacers and the channel geometry have once again presented in this paper for the readers convenient.

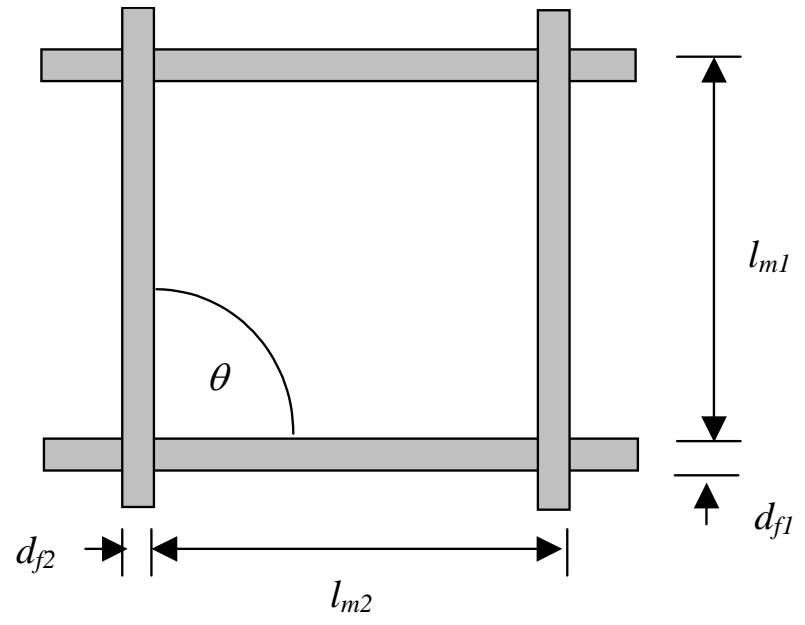

Figure 1. Definition of spacer dimensions. [19]

Spacers come in various forms that are comprised of a net-like arrangement of filaments aligned parallel, transverse or at an angle to the module axis. Different diameters, various mesh lengths are observed in commercially available spacers. In the present work,flows in narrow channels with cylindrical obstructions is investigated to improve fundamental understanding of mass transfer. In order to elucidate the likely extent of flow disturbance, the CFD simulations focused on the effect of cylindrical obstructions positioned normal to the flow. This corresponds to transverse filaments, and allows two-dimensional simulations. Three arrangements are simulated as shown in Fig (2).

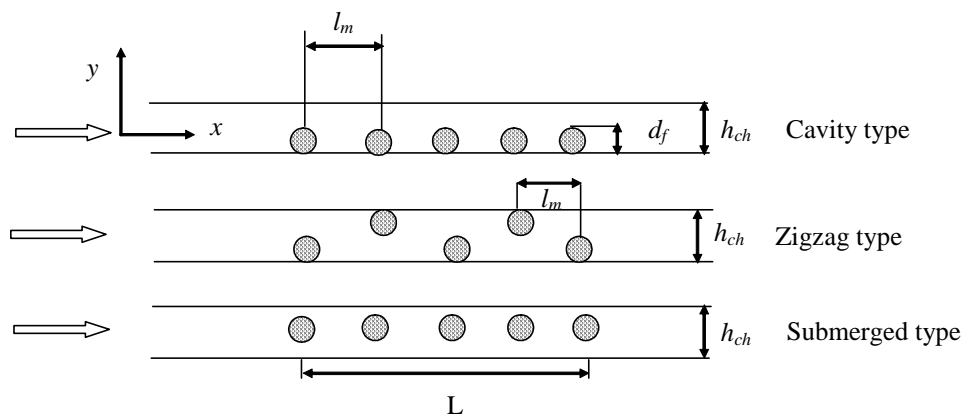

Figure 2. Various arrangements of Transverse filaments 
(i) Simulation of spacers touching the same channel wall, (ii) Simulation of spacer cylinders touching alternately adjacent to the top and bottom wall, (iii) Simulations of spacer cylinders immersed in the channel. The computational domain is set up with a channel entrance length of 10 times the filament diameter, (see Figure 3) while the exit length is at least twice the entrance length to avoid any effects of the channel exit on eddy formation behind the filaments. The inlet velocity is specified uniform and as normal to the channel entrance and the flow is fully developed before reaching the first upstream filament. The wall is assumed to be non permeable to analyze the hydrodynamics between two sequential filaments because permeate recoveries are usually low for membrane applications. All simulated channel configurations had an identical channel height. Parametric studies of the dimensionless mesh length $l_{m} / h_{c h}$, the channel Reynolds number $R e_{c h}$ are performed to cover the range of spacer dimensions used in commercial spiral-wound membrane modules and in other studies examining spacers. The channel Reynolds number $R e_{c h}$ is defined as: $\operatorname{Re}_{c h}=d_{h} u_{\text {ave }} / v$ where $d_{h}$ is the hydraulic diameter for spacer filled flow channel. A schematic of the flow channel is presented in Fig. 3.

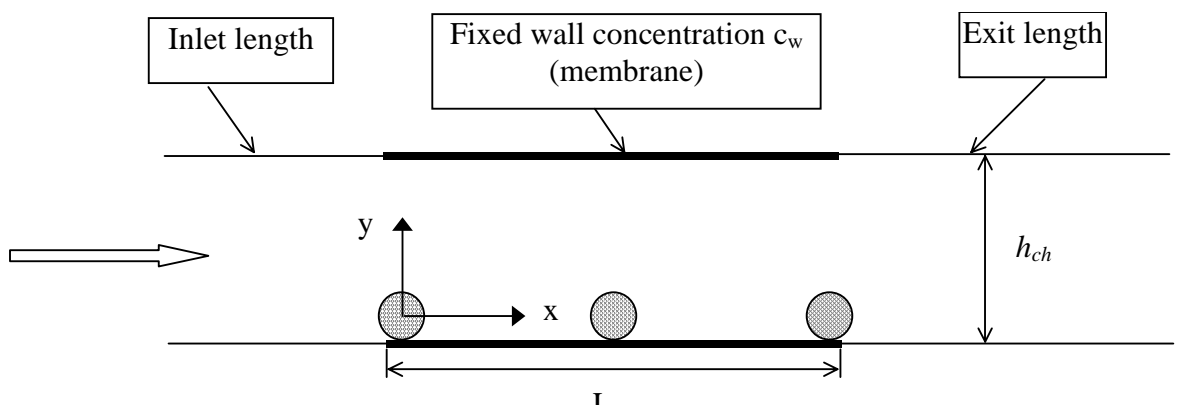

L

Figure 3. Channel geometry and flow configuration

\section{Numerical Method and Modeling assumptions:}

Simulations were performed over a range of channel Reynolds numbers $\left(\operatorname{Re}_{c h}=d_{h} u_{\text {ave }} / v\right) 100-400$. The fluid is taken as water at a temperature of $293 \mathrm{~K}$ and it is assumed to be incompressible and isothermal and to have constant fluid properties. The general Navier-Stokes equations governing the flow field are solved by using a commercial CFD package FLUENT 6.0. The code utilizes a collocated finite volume formulations, and second order upwind differencing scheme is used in all simulations. The equations are solved by using the SIMPLE algorithm with an iterative line-by-line matrix solver. For low Reynolds numbers the flow remains stationary and no vortex shedding is produced. Convergence is achieved in less than 2000 iterations. An unstructured grid with sufficiently fine resolution is chosen to ensure that the results are independent of grid size. The number of cells generated depended on the number of filaments used for the simulation. 


\section{Mass Transfer on the spacer filled narrow channel:}

\subsection{Modeling assumption:}

Mass transfer enhancement on the spacer filled channel is investigated in this present work. Channel geometry and configuration of the spacers are exactly the same as those taken for studying the Hydrodynamics of the narrow channel filled with net type cylindrical spacers[1],[2]. Since the membrane wall reject the solutes, while solvent can pass through it, the average bulk concentration along the channel increases. Thus concentration polarization occurs. During the present simulations, a fixed higher value of the wall concentration along the mass transfer region, $\mathrm{L}$, is taken in order to simulate the concentration polarization. This simplified approach of simulating the concentration polarization is suitable for many practical applications because a gel layer of fixed high osmotic pressure or viscosity is formed, so a limiting value of concentration at the wall is reached. It is also assumed that the walls are non permeable and the velocity at the walls are assigned as zero.

Simulations are carried out for the whole computational domain and the channel height and the mass transfer region is kept constant for all types of filament configurations. Channel Reynolds number, based on the hydraulic diameter of the spacer ranges from 100-400, corresponding to the steady regimes discussed earlier. For illustrating the effects of the dimensionless mesh length, $l_{m} / h_{c h}$, the number of filaments on the mass transfer region is adjusted with constant, L. The exit region is taken long enough so that any interference between the channel exit and the concentration profile formed behind the last downstream filament is avoided. All the assumptions for the flow field are exactly similar to those stated earlier for the Hydrodynamics simulations. Another scalar transport equation with convection-diffusion terms is included with the governing transport equation:

$$
u \frac{\partial c}{\partial x}+v \frac{\partial c}{\partial y}=D\left(\frac{\partial^{2} c}{\partial x^{2}}+\frac{\partial^{2} c}{\partial y^{2}}\right)
$$

In eqn. (1) $D$ is the diffusion coefficient. The diffusivity and the density are considered as constant. For predicting the concentration profile, the Schmidt number, $S c=v / D$ is taken as 100. [7].

\subsection{Concentration profile in Narrow channels with transverse filaments:}

Concentration profiles for single filament adjacent to the bottom wall and submerged in the center of the channel are presented in Figure $4(a, b)$. It was shown earlier that for single filament adjacent to the bottom wall a small recirculation region forms in front of the filament and a large one forms behind the filament. Because of the formation of these recirculation regions, the concentration boundary layer is disturbed at both the top and bottom wall. As a result of the flow recirculation, concentration within the recirculation region is increased near the bottom wall. Higher velocity at the top of the filament and higher value of wall shear stress disturb the formation of the concentration boundary layer at the top wall. 
For a spacer submerged in the center of the channel, concentration boundary layer at both top and bottom wall are altered because of the velocity acceleration as well as the formation of the secondary flows. Higher velocity at top and bottom of the filaments leads to an enhancement of wall shear stress at both walls, which also results in a change of near wall scalar transport.

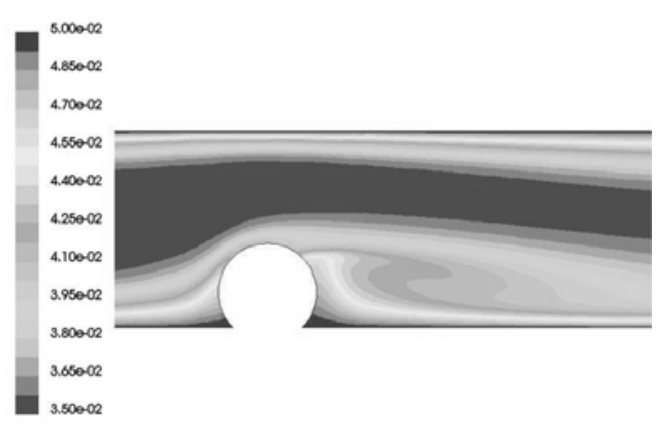

(a)

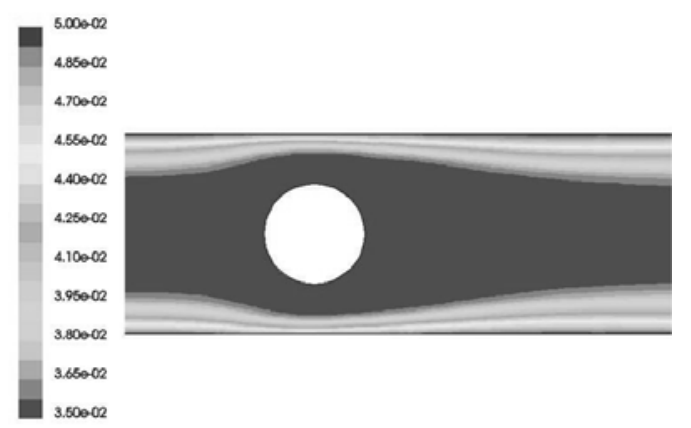

(b)

Figure 4(a,b): Concentration profiles (a) for a single filament adjacent to the bottom wall, (b) for a single filament submerged in the center of the channel, $R e_{c h}=300, S c=100, d_{f} / h_{c h}=0.5$.

\subsection{Concentration profiles in the narrow channel with multiple filaments:}

The concentration field is simulated for the three different above mentioned configurations of the spacers. For the cavity spacers, when the dimensionless mesh length, $l_{m} / h_{c h}$ is lower (i.e.3), as in Fig.5 (a) a large recirculation region is formed between sequential filaments. Thus, concentration within this recirculation region gets higher than the bulk concentration. Similar to the single filament placed adjacent to the bottom wall, the concentration boundary layer is disturbed above the filament because of the higher velocity and higher absolute value of shear stress. When the dimensionless mesh length is higher (i.e 6), as in Fig 5 (b), separate recirculation regions form between sequential filaments and the separation streamlines of the recirculation region reattach to the wall. Hence concentration profile shows the same manner as single filament adjacent to the bottom wall.

Figure 6(a) shows that, for the submerged spacers, at $R e_{c h}=300, S_{c}=100, d_{f} / h_{c h}=0.5$ and $l_{m} / h_{c h}=3$, the concentration boundary layer on top and bottom wall is altered in a similar fashion to a single filament submerged in the center of the channel. Since higher velocities arises on top and bottom of each filament, the wall shear stress also becomes higher and disturbance of the formation of concentration boundary layer is enhanced.

For the spacers, placed alternately top and bottom of the wall, the concentration increases behind each of the filaments because of the formation of recirculation region, as shown in

Figure 6 (b). Development of concentration boundary layer on the wall opposite each of the filaments is disturbed by the higher velocity and shear stress. 


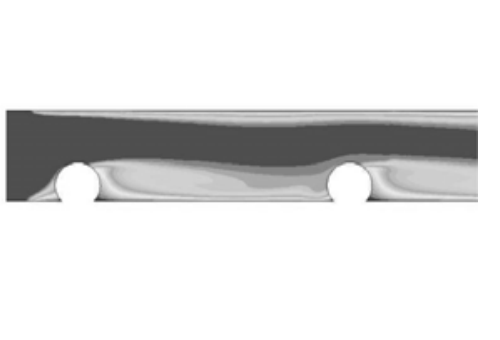

(a)

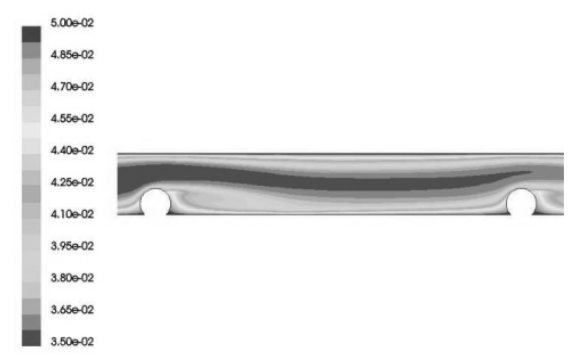

(b)

Figure 5 (a,b): Concentration profiles (a) for cavity spacer with $l_{m} / h_{c h}=3$, (b) for cavity spacers with $l_{m} / h_{c h}$ $=6, R_{c h}=300, S_{c}=100, d_{f} / h_{c h}=0.5$.
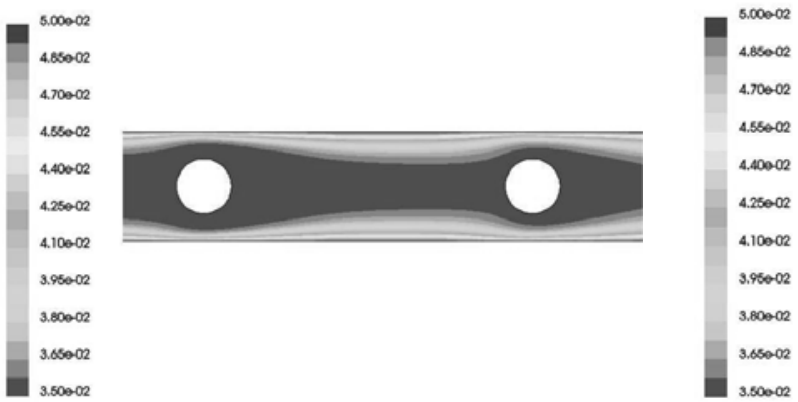

(a)

(b)

Figure 6 (a,b): Concentration profiles (a) for submerged spacers with $l_{m} / h_{c h}=3$, (b) for zigzag spacers with $l_{m} / h_{c h}=6, R e_{c h}=300, S c=100, d_{f} / h_{c h}=0.5$.

\subsection{Mass transfer Enhancement in Spacer-Filled Channel:}

In order to estimate the mass transfer enhancement, the efficiency of the spacer is calculated by:

$$
\eta_{m t}=\frac{\Delta c_{\text {spacer }}}{\Delta c_{\text {slit }}}
$$

where $\eta_{m t}$ is the efficiency of the spacer and $\Delta c_{\text {spacer }}$ and $\Delta c_{\text {slit }}$ are the concentration increase along the spacer-filled channel and the slit (empty channel), respectively. Calculations are performed for an empty channel (slit) to obtain the value of $\Delta c_{\text {slit }}$. Figure

7 (a,b) shows the mass transfer enhancement pattern and pressure loss ratio for the cavity spacer with channel Reynolds number 300 for different dimensionless mesh lengths $l_{m} / h_{c h}$. As the mesh length decreases from 8 , the mass transfer enhancement, $\eta_{m t}$ increases and a local maximum at $l_{m} / h_{c h}=4$ of $\eta_{m t, m a x} \approx 1.65$ is obtained. Further decrease of mesh length leads to a continuous reduction of the mass transfer enhancement, $\eta_{m t}$. For larger mesh length at $l_{m} / h_{c h}=8$ and above, a closed recirculation region exists. As long as this recirculation region is maintained, mass transfer enhancement increases with reduction of 
the mesh length. For the mesh length $l_{m} / h_{c h}=4$, a fully formed recirculation region is observed between sequential filaments and a local maximum value of mass transfer enhancement, $\eta_{m t}$ is obtained. When the mesh length is reduced further, the size of the fully formed recirculation region become smaller. This is because, the filaments obscure a higher amount of wall area which results into the reduction of the over all mass transfer enhancement.

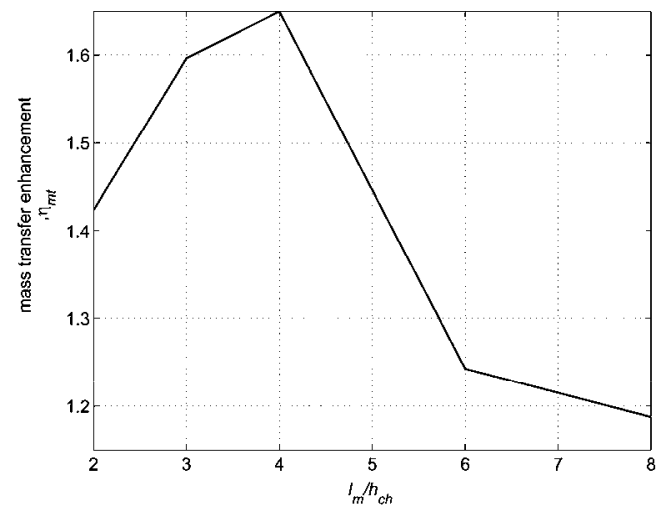

(a)

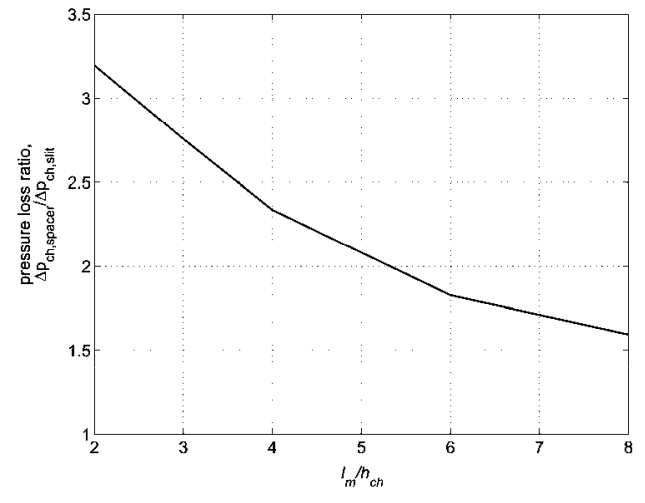

(b)

Figure 7: (a) mass transfer enhancement (b) pressure loss along th channel, for the cavity spacer at different mesh length with channel Reynolds number $R_{c h}=300, S c=100$

Mass transfer enhancement patterns and pressure loss ratio for the submerged spacer for different mesh length is shown in Figure 8 (a,b). For the submerged spacer, mass transfer enhancement, $\eta_{m t}$, increases monotonically as the mesh length decreases until $l_{m} / h_{c h}=2$ and no local maximum occurs as in the preceding case. For the zigzag spacer Fig. 9 (a ,b), similar to the submerged spacer, the mass transfer enhancement increases as the dimensionless mesh length decreases. Higher number of filaments cause mass transfer enhancement.

Figure 10 (a,b) compares the mass transfer enhancement and pressure loss for different types of filament configuration. In order to make comparison of the mass transfer enhancements of the three different types of spacers, simulations are carried out with a constant concentration for both top and bottom wall and Channel Reynolds number is taken as 200. From the figure it can be observed that, submerged spacer shows the highest mass transfer enhancement. Pressure loss along the channel is also greatest for the submerged spacers. All types of spacers shows higher mass transfer enhancement with the decrease of dimensionless mesh length. For the cavity spacers, the mass transfer enhancement is lowest for the same mesh length compare to submerged and zigzag spacers. Pressure loss along the channel is also lowest for the cavity spacers than the zigzag and submerged spacers. 


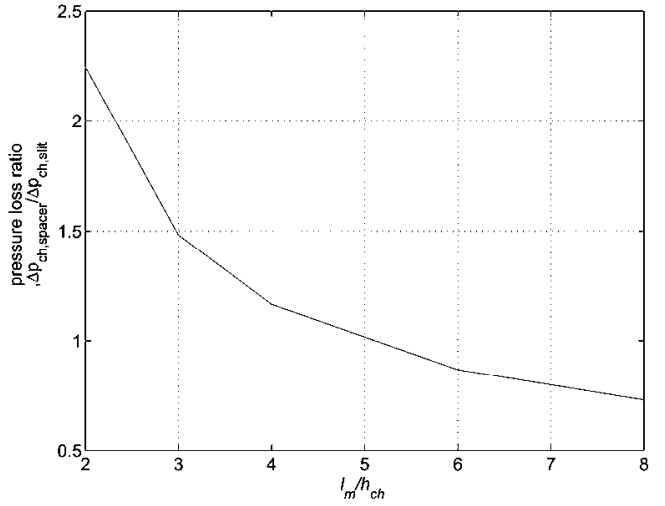

(a)

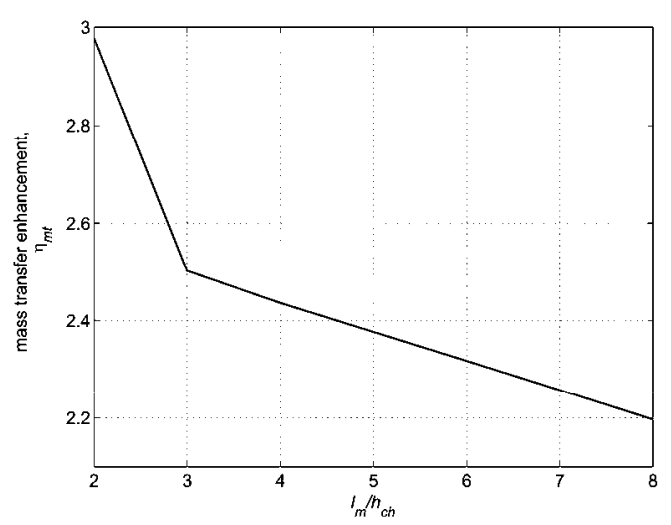

(b)

Figure 8: (a) mass transfer enhancement (b) pressure loss along the channel, for submerged spacer at different meshlength with channel reynolds number $R e_{c h}=300$ and $S_{c}=100$

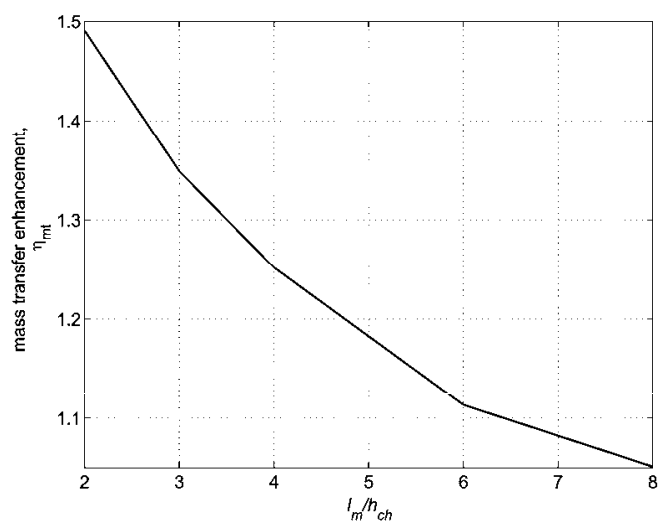

(a)

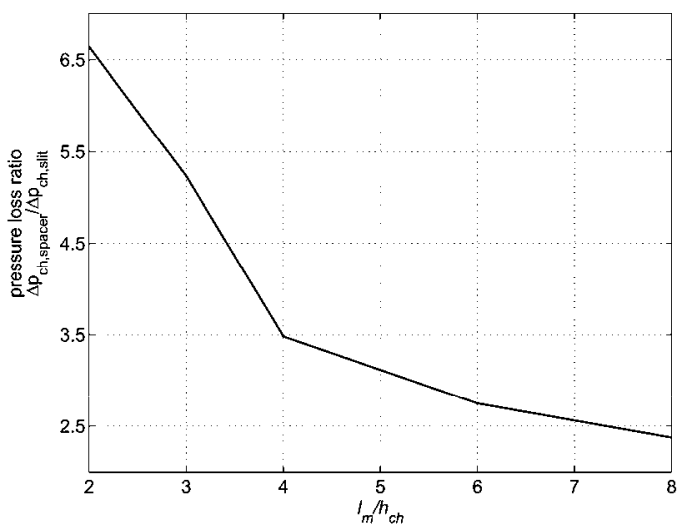

(b)

Figure 9: (a) mass transfer enhancement (b) pressure loss ratio along the channel, for zig zag spacer at different mesh length with channel Reynolds number, $R e_{c h}=300$ and $S c=100$

\section{Conclusion}

This study presents CFD simulations mass transfer in spacer filled membrane module. Laminar flow is first considered and fixed wall concentration boundary condition is used to simulate the concentration polarization for the concentration field. Calculations are carried out for two dimensional channel geometry. Reduction of fouling and concentration polarization by modification of hydrodynamic condition is very important for enhancing the membrane module performances. Investigation of the flow pattern in a spacer filled channel have revealed the importance of hydrodynamics on mass transfer and pressure loss enhancement along the flow channel. An effective spacer should provide higher mass transfer rate from the membrane wall to the bulk flow in order to reduce the concentration polarization with maintaining lower pressure loss along the channel. Among the three types of spacers, submerged spacers show the highest wall 
shear stress and mass transfer enhancement though this types of spacers results in the largest pressure loss along the channel.

Cavity types of spacers show the lowest performance. The presence of spacers in the channel results in local concentration maximum which exceeds the corresponding concentration in the case of empty channel. From the analysis of mass transfer, it can be observed a complex correlation among the flow pattern, wall shear stress, mass transfer enhancement, pressure loss along the channel being dependent on the channel Reynolds number, mesh length and spacer configuration.

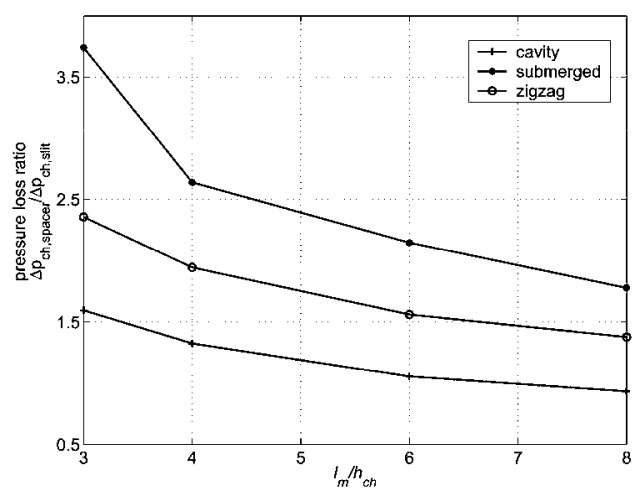

(a)

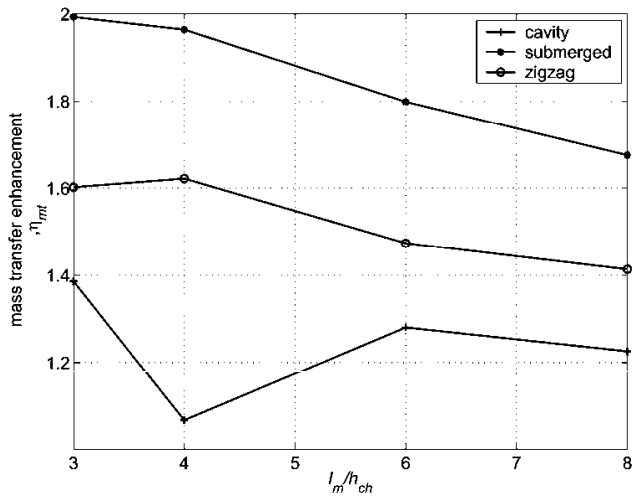

(b)

Figure 10: comparison of the (a) mass transfer enhancement (b) pressure loss along the channel, at different mesh length for three types of spacers with channel Reynolds number $\operatorname{Re}_{c h}=200, S_{c}=100$.

\section{REFERENCES}

1. Hussain, Sharmina ., Computational fluid dynamics study of flow behaviour in a single spacer filled membrane module, BRAC University Journal, Vol.5, No.1, 2008.

2. Hussain, Sharmina, Computational fluid dynamics study of flow behaviour in membrane module with multiple filaments, BRAC University Journal, Vol.6, No.2, 2009.

3. Srikanth, G., Membrane separation Processes-Technology and Business opportunities, http://www.tifac.org.in/news/memb.htm, 31'st January, 2004.

4. Schwinge, J., D. E.Wiley, Fletcher D.F, Simulation of the flow around spacer filaments between channel Walls.2. Mass transfer. Ind. Eng. Chem. Res. 41, 4879-4888, 2002.

5. Schwinge, J., Wiley, D. E., Fane, A.G., Novel spacer design improves spiral wound module performance, Proc. $6^{\text {th }}$ World Congress of Chemical Engineering, Melbourne, Australia, 2001.

6. Schwinge, J., Wiley, D. E. , Fletcher D. F., A CFD study of unsteady flow in narrow spacer filled channels for spiral-wound membrane modules, Desalination, 195-201, 2002

7. Schwinge, J., Wiley, D.E., Fletcher, D.F., Simulation of the flow around spacer filaments between narrow channel Walls.1. Hydrodynamics. Ind. Eng. Chem. Res. 41, 2977-2987, 2002.

8. Wiley, Dianne. E., Fletcher, D. F., Techniques for computational fluid dynamics modeling of flow in membrane channels, Journal of Membrane science, 211, 127-137, 2003.

9. Wiley, Dianne. E., Fletcher D. F., Computational fluid dynamics modeling of flow and permeation for pressure-driven membrane processes, Desalination, 145, 183-186, 2002. 